\title{
Sleep and Respiration of Perry Syndrome: Polysomnographic and Pathological Correlations
}

\author{
Ki-Hwan Ji, MD, Mi-Ri Kang, MD, Eun Joo Chung, MD, Sang Jin Kim, MD \\ Department of Neurology, Busan Paik Hospital, Inje University College of Medicine, Busan, Korea
}

Received: June 1, 2018

Revised: June 19, 2018

Accepted: October 10, 2018

Correspondence

Ki-Hwan Ji, MD

Department of Neurology,

Busan Paik Hospital,

Inje University College of Medicine,

75 Bokji-ro, Busanjin-gu,

Busan 47392, Korea

Tel +82-51-890-8613

Fax +82-51-890-6130

E-mail kihwanji@gmail.com

ORCID

Ki-Hwan Ji

https://orcid.org/0000-0002-5371-5398

Mi-Ri Kang

https://orcid.org/0000-0002-9833-023X

Eun Joo Chung

https://orcid.org/0000-0001-8948-1135

Sang Jin Kim

https://orcid.org/0000-0001-7240-2154
Perry syndrome is a rare, adult-onset, autosomal dominant, progressive neurodegenerative disorder that is characterized by parkinsonism, weight loss, psychiatric symptoms, and central hypoventilation. We report the case of a 51-year-old man, whose condition was genetically identified as Perry syndrome. Here we focus the polysomnographic (PSG) findings and discuss the correlations between PSG and neuropathological findings.

Sleep Med Res 2018;9(2):115-117

Key Words Polysomnography, Perry syndrome, Cheyne-Stokes respiration, Hypoventilation, Neuropathology.

\section{INTRODUCTION}

Perry syndrome is a rare autosomal-dominant disease characterized by early-onset parkinsonism, weight loss, depression, psychiatric symptoms and central hypoventilation $[1,2]$. The diagnostic criteria of Perry syndrome are suggested as 1) parkinsonism, family history plus mutation in the exon 2 of dynactin 1 (DCTN1) gene, or 2) parkinsonism, psychiatric symptoms, and respiratory symptoms plus TAR DNA-binding protein (TDP-43) pathology [3]. Herein, we report a 51-year-old man with advanced parkinsonism (the Unified Parkinson's Disease Rating Scale 63 and Hoehn \& Yahr stage 5). 18F-fluorinated N-3-fluropropyl-2 $\beta$ carboxymetoxy-3 $\beta$-nortropane positron emission tomography showed a marked loss of dopamine transporters. Findings of DCTN1 mutation supported his diagnosis. The autopsy revealed TDP-43 pathology. As the sleep features of Perry syndrome are mostly unknown, we focus on the polysomnographic (PSG) findings and further discuss the correlations between PSG and neuropathological findings.

\section{CASE REPORT}

\section{Polysomnographic Findings}

Although extensive interviews were conducted with the patient's family members, they knew little about his sleep-wake patterns. He was completely bed-ridden when he took the PSG exam. Therefore, we studied the patient's sleep patterns using PSG from 17:00 to the next day 11:00 (Fig. 1).

While waking, an arterial blood gas analysis indicated mild hypercapnia without hypoxemia ( $\mathrm{pH}, 7.42, \mathrm{PcO}_{2}, 51 \mathrm{~mm} \mathrm{Hg}, \mathrm{HCO}_{3}^{-}, 32.5 \mathrm{~mm} \mathrm{Hg}, \mathrm{Po}_{2}, 91.7 \mathrm{~mm} \mathrm{Hg}$ ). Cheyne-Stokes respiration (CSR), the periodic breathing pattern in which the tidal volume gradually waxes and wanes in a crescendo-decrescendo manner, was evident. He slept 248.5 min, and two distinct periods of sleep, from 21:00 to 22:00 and from 02:00 to 06:00, were identified. The proportion of N1 was elevated to $41.6 \%$ (normal range of $1-5 \%$ ) and $\mathrm{N} 2$ was reduced to $22.2 \%$ (normal range of 
40-50\%). Deep sleep was absent. The proportion of REM was $26.2 \%$ which was in the normal range. While sleeping, the patient experienced semi-periodic breathing, although the breathing was discrepant from CSR. He had apneas with obstructive apnea index of $11.4 / \mathrm{h}$, hypopnea index of 33.6/h. Most hypopneas might be classified as central because hypopneas were not associated with snoring, thoracoabdominal paradox, and increased inspiratory flattening of the nasal pressure flows signal during events [4]. Hypoxemia was mild (average oxygen saturation, $92.6 \%$; desaturation to the nadir of $87 \%$ ). The arousal index was elevated to $35 / \mathrm{h}$, and three-quarters of arousals occurred with respiratory events. Abnormal leg movements were absent.

\section{Neuropathological Findings}

The autopsy revealed an almost complete neuronal loss in the substantia nigra, reactive gliosis, and microvacuolation. There was also some neuronal loss in the globus pallidus, subthalamic nucleus, thalami and brainstem nuclei. Moderate neuronal loss was presented in the locus coeruleus and inferior olivary nucleus, and mild loss was presented in the pontine tegmentum. Anti-phospho-TDP-43 antibodies reacted to surviving neurons.

\section{DISCUSSION}

PSG revealed irregular sleep-wake cycles, although the patient showed a nocturnal sleeping tendency. We can summarize the significant findings from PSG as follows; 1) highly fragmented and altered sleep architectures: disturbed nonREM (increased N1 and absent N3) but relatively preserved proportion of REM sleep, 2) abnormal breathing: hypoventilation and CSR while waking, and semi-periodic breathings similar to CSR, and obstructive sleep apnea mainly composed of central hypopnea while sleeping. Among the late symptoms of Perry syndrome, hypoventilation is the most dangerous because it may lead to respiratory failure and death [1]. Most patients with Perry syndrome have rapid, shallow breathing that alternates with normal breathing, hypopnea, or apnea [2].

The neuropathological findings are consistent with earlier reports of TDP-43 proteinopathy [5]. Complete neuronal loss in the substantia nigra was observed. The brainstem, especially the midbrain and pons, was the site of preferential neuronal loss.

Dopaminergic modulation of sleep-wake behaviors has been postulated: Dopamine promotes sleep through the basal ganglia networks but also promotes wakefulness through extrabasal ganglia circuits [6]. Besides respiratory disturbances, dopaminergic dysfunction through wide-spread neuronal loss in the substantia nigra, globus pallidus, subthalamic nucleus, and thalamus may contribute to the disrupted sleep-wake cycles of the patient. Meanwhile, the pedunculopontine nucleus, where cholinergic neurons reside in the pontine tegmentum, is involved in REM generation and maintenance [7]. Mild neuronal loss in that area might be associated with relatively preserved REM sleep.

The respiratory center is located in the medulla and pons. In this case, moderate neuronal loss presented in the locus coeruleus of the pons and inferior olivary nucleus of the medulla. In the medulla, the ventral groups of respiratory neurons (VRG) generate respiration, thus the bilateral VRG lesions caused a loss of automated breathing [8]. The dorsal raphe nucleus located on the midline of the midbrain and pons, which is sensitive to hypercapnia, projects to the VRG via serotonin [2]. An autopsy report had demonstrated the neuronal loss in the ventrolateral medulla and dorsal raphe nucleus in Perry syndrome [9]. CSR caused by brain stem structural lesions is also well known [10]. The impaired respiratory center located in the medulla and pons can be the neuropathological substrate of hypoventilation and CSR in Perry syndrome.

The patient had a tracheostomy. Six months later, he had a sudden unexpected death, which is frequently seen in Perry

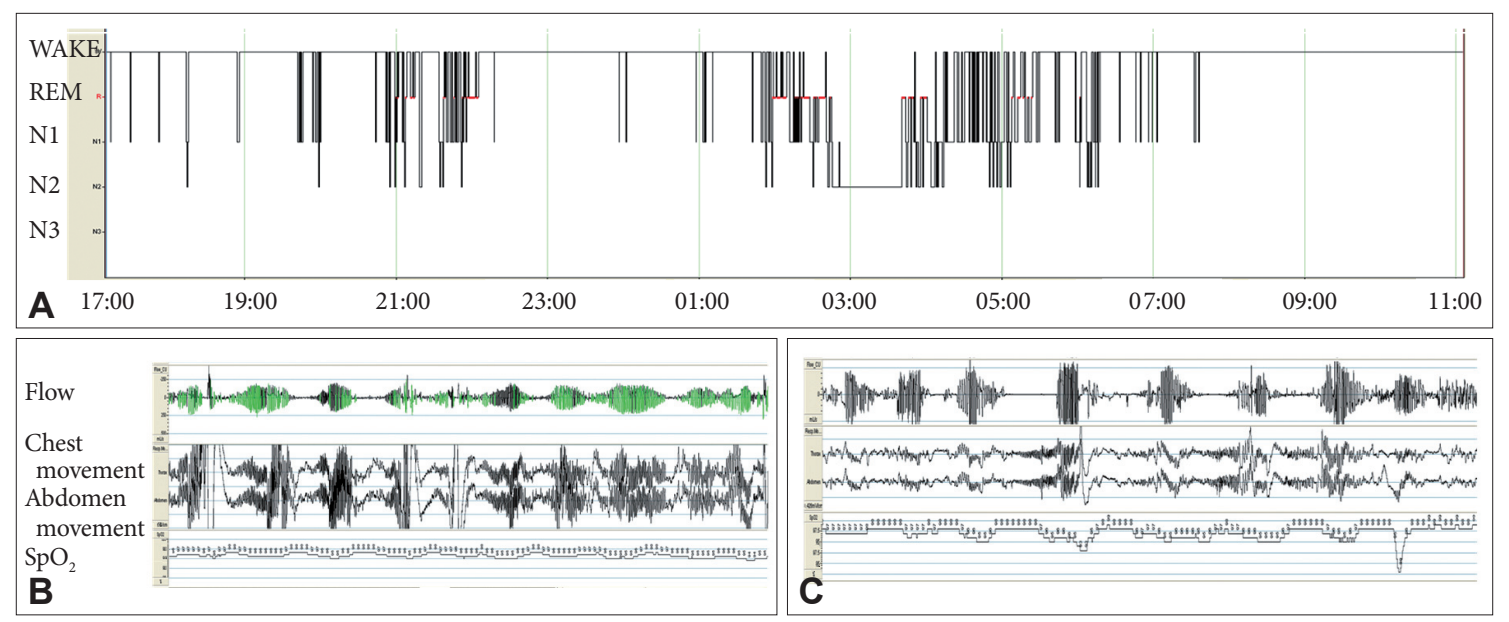

Fig. 1. Hypnogram and 600-second excerpts. A: Hypnogram revealed sleep fragmentation, loss of sleep continuity and absent deep sleep. B: A 600-second excerpt showed the classic Cheyne-Stokes respiration crescendo-decrescendo pattern in wakefulness. C: A 600-second excerpt demonstrated the prolonged periodic apnea or hypopnea associated with oxygen desaturations during REM. 
syndrome [1]. As a single-patient trial, a patient showing mild extrapyramidal symptoms but severe respiratory failure with Perry syndrome was inserted with a diaphragmatic pacemaker, and patient's respiratory insufficiency was controlled [11]. However, the proper management of respiratory symptoms of Perry syndrome is unknown.

\section{Conflicts of Interest}

The authors have no financial conflicts of interest.

\section{Authors' Contribution}

Conceptualization: Ji KH, Kim SJ. Resources: Chung EJ, Ji KH. Writingoriginal draft: Ji KH. Writing_review \& editing: Ji KH, Kang MR, Chung EJ, Kim SJ.

\section{REFERENCES}

1. Ohshima S, Tsuboi Y, Yamamoto A, Kawakami M, Farrer MJ, Kira J, et al. Autonomic failures in Perry syndrome with DCTN1 mutation. Parkinsonism Relat Disord 2010;16:612-4.

2. Wider C, Wszolek ZK. Rapidly progressive familial parkinsonism with central hypoventilation, depression and weight loss (Perry syndrome)--a literature review. Parkinsonism Relat Disord 2008;14:1-7.

3. Mishima T, Fujioka S, Tomiyama H, Yabe I, Kurisaki R, Fujii N, et al. Establishing diagnostic criteria for Perry syndrome. J Neurol Neurosurg
Psychiatry 2018;89:482-7.

4. Berry RB, Budhiraja R, Gottlieb DJ, Gozal D, Iber C, Kapur VK, et al. Rules for scoring respiratory events in sleep: update of the 2007 AASM Manual for the Scoring of Sleep and Associated Events. Deliberations of the Sleep Apnea Definitions Task Force of the American Academy of Sleep Medicine. J Clin Sleep Med 2012;8:597-619.

5. Wider C, Dickson DW, Stoessl AJ, Tsuboi Y, Chapon F, Gutmann L, et al. Pallidonigral TDP-43 pathology in Perry syndrome. Parkinsonism Relat Disord 2009;15:281-6.

6. Vetrivelan R, Qiu MH, Chang C, Lu J. Role of Basal Ganglia in sleepwake regulation: neural circuitry and clinical significance. Front Neuroanat 2010;4:145.

7. Romigi A, Placidi F, Peppe A, Pierantozzi M, Izzi F, Brusa L, et al. Pedunculopontine nucleus stimulation influences REM sleep in Parkinson's disease. Eur J Neurol 2008;15:e64-5.

8. Zigmond MJ, Hastings TG, Perez RG. Increased dopamine turnover after partial loss of dopaminergic neurons: compensation or toxicity? Parkinsonism Relat Disord 2002;8:389-93.

9. Tsuboi Y, Dickson DW, Nabeshima K, Schmeichel AM, Wszolek ZK, Yamada T, et al. Neurodegeneration involving putative respiratory neurons in Perry syndrome. Acta Neuropathol 2008;115:263-8.

10. Lee MC, Klassen AC, Heaney LM, Resch JA. Respiratory rate and pattern disturbances in acute brain stem infarction. Stroke 1976;7:382-5.

11. Pretelt F, Castañeda Cardona C, Tacik P, Ross OA, Wszolek ZK. Latin America's first case of Perry syndrome and a new treatment option for respiratory insufficiency. J Neurol 2014;261:620-1. 